\title{
MIPAS-Family - evaluation of a new multi-modal behavioral training program for pediatric headaches: clinical effects and the impact on quality of life
}

\author{
Wolf-Dieter Gerber • Franz Petermann • Gabriele Gerber-von Müller • \\ Maren Dollwet · Stephanie Darabaneanu $\cdot$ Uwe Niederberger · \\ Ilva Elena Schulte · Ulrich Stephani · Frank Andrasik
}

Received: 7 October 2009/Accepted: 12 January 2010/Published online: 8 April 2010

(C) Springer-Verlag 2010

\begin{abstract}
Several meta-analyses have demonstrated that the combination of electrical muscle activity and Temperature Biofeedback could be regarded as gold standard in chronic pediatric headaches. However, these techniques seem to be uneconomical and furthermore they are not directed to improve the social competence as well as resolve possible impairments in daily activities of the child. Therefore, multi-modal behavioral techniques have been proposed, but no studies comparing these with the gold standard were conducted. The present study compared the impact of a new multi-modal behavioral education and training program-MIPAS-Family—with a combined Biofeedback treatment, evaluating clinical efficacy as well as the effect on the quality of life (QoL) of children with chronic headaches. Thirty-four children and adolescents with recurrent headache, ranging from 7 to 16 years, were randomly assigned to the MIPAS-Family $(N=19)$ or the Biofeedback $(N=15)$ condition. All patients were diagnosed by the criteria of the International Headache Society. The children and their parents completed headache diaries,
\end{abstract}

W.-D. Gerber $(\bowtie) \cdot$ G. Gerber-von Müller · S. Darabaneanu U. Niederberger

The Institute of Medical Psychology and Medical Sociology, University of Kiel, 24113 Kiel, Germany

e-mail: gerber@med-psych.uni-kiel.de

\section{F. Petermann · I. E. Schulte}

The Center for Clinical Psychology and Rehabilitation, University of Bremen, Bremen, Germany

M. Dollwet · F. Andrasik

The Center of Applied Psychology, University of West Florida, Pensacola, FL, USA

\section{U. Stephani}

Neuropediatric Hospital, University of Kiel, Kiel, Germany diaries of daily living activities and a QoL questionnaire $\left(\mathrm{KINDL}^{\circledR}\right)$. Both groups showed significant improvements concerning the headache intensity and headache duration. We found no significant differences in the main headache parameters between both treatments. After the treatments, the children were less disturbed by their headaches in the domains school, homework, and leisure time. In conclusion, MIPAS-Family is as effective as Biofeedback but it is more cost-effective and addresses the whole family and the daily activities.

Keywords Pediatric headaches - Biofeedback · MIPAS-Family Program $\cdot$ Education $\cdot$ Behavioral medicine

\section{Introduction}

Nearly $70 \%$ of children and adolescents report occasional headaches, with $20 \%$ suffering from chronic headaches that are mainly migraine and tension-type. The prevalence of pediatric headaches has increased significantly during the last 30 years in western countries as well as in Asia [1-4]. To compound matters, these headache conditions often have significant negative social and economic impacts [57]. For example, several studies using impairment scales (PedMIDAS: Pediatric Migraine Disability Assessment) or quality of life (QoL) scales (PedsQL 4.0; Paediatric Quality of Life Inventory) have ascertained that children suffering from headaches show a considerable reduction in school attendance and leisure activities when compared to healthy children [2, 8-15]. Frare et al. [10] emphasized that the headache frequency and duration have a significant impact on a child's QoL and that the family daily routine, how the family members adapt to the child's pain, influences significantly both the child's coping ability and the child's 
QoL. On top of this, these children have a higher risk for anxiety disorders and depression [16].

Behavioral medicine approaches, particularly Biofeedback, relaxation training, as well as cognitive-behavioral techniques have been identified as efficacious treatments for headache, both for adults and children [17-25]. Usually these treatments are applied somewhat in isolation and involve therapy contact with the patient alone. One exception is the "Stop the Headache" program of KrönerHerwig and Dencke [26, 27], which added parent education to a specific stress management training (SMT) program. Based on their encouraging findings and results from family interaction studies and biopsychological investigations of migraine, we developed a multi-modal behavioral educational group program that included specific training for children and their parents [28-30]. This so-called Migraine-Patient-Seminar Program (MIPAS-Family) has the advantage of incorporating the daily living activities of the children and the whole family as well as potentially reducing the financial costs of individual behavioral treatment.

The present study thus aimed to compare the clinical efficacy of this new multi-modal behavioral education program for children with headaches and their parents. This program was in turn compared to a "benchmark" treatment-Biofeedback. A further aim was to test for improvements in non-pain measures, exploring whether one or both treatment conditions, above the influence of headache parameters, have a positive impact on the child's QoL and the child's ability to take part in daily living activities.

\section{Methods}

\section{Patients}

Sixty (60) consecutive children and adolescents, ranging from 7 to 16 years of age, and their parents were considered for participation in the study. They were termed eligible if they suffered from migraine without aura, tension-type headache, or the combination of both. The patients were recruited mainly from the Clinic of Neuropediatrics of the University Hospital of Kiel. All patients received neurological and clinical examinations concerning the headache diagnosis. Exclusion criteria were the presence of chronic daily headache ( $\geq 15$ days per month) or drug abuse. Forty (40) patients fulfilled the inclusion criteria of the International Headache Society (IHS) classification and were randomized (by age and sex) to one of the two intervention groups. Five children dropped out during the pre-observation phase because of lack of compliance (one child from the
MIPAS-Family group and four children from the Biofeedback group).

Data were thus available from 34 children $(N=19$, $68 \%$ girls and $32 \%$ boys for the MIPAS-Family group; $N=15,60 \%$ girls and $40 \%$ boys for the Biofeedback group). The mean age was 11.16 years $(\mathrm{SD}=2.75$, range 7-16) for the MIPAS-Family group and 12.53 years ( $\mathrm{SD}=2.03$, range 9-16) for the Biofeedback group. The mean percentages of headache frequency prior to treatment were $56.2 \% 2$ days per the week, $21.1 \% 1$ day per week and $23.7 \%$ 1-3 days per month for the MIPAS-Family group, and 40\% 2 days per week, $33.3 \% 1$ day per week and $27.3 \%$ 1-3 days per month for the Biofeedback group. Mean headache duration per week in the Biofeedback group was higher $(M=11.88 \mathrm{~h}, \mathrm{SD}=10.20)$ than in the MIPAS-Family group $(M=4.59 \mathrm{~h}, \mathrm{SD}=2.91)$. In the MIPAS condition, $47.4 \%$ of the children suffered from migraine and $52.6 \%$ from combination headache, while $33.3 \%$ of the Biofeedback group had migraine and $66.7 \%$ combination headaches. No child fulfilled the criteria of pure TTH. Both groups did not differ significantly in the most variables except for variable statements of the parents concerning headache frequency. Three children in each condition could not be reached at the 1-year follow-up.

\section{Procedure}

Once cleared for the project, parents and their children were informed of the aims of the two intervention programs and then they were asked for their statement of agreement. Both parties completed headache questionnaires and were asked to begin completing headache diaries (for 4 weeks, baseline). They were then randomized to one of the two conditions. These measures were recompleted 6 and 12 months following completion of treatment, in person when possible. The headache diaries have been completed 4 weeks during the pre-observation and during the whole treatment period. For families who were not able to visit our institute personally these measures were collected by the postal system. The ethical committee at the University of Kiel granted permission for this study.

Interventions

\section{Description of the MIPAS-Family Program}

The MIPAS-Family Program consisted of two main components: child training (8 sessions, $90 \mathrm{~min}$ each; see Table 1) and parent training (4 sessions, 120 min each; see Table 2). The sessions were divided into three modulesdiagnostic, education, and behavioral training. The child and parent components were alternated (1 week child training and the other week parent training) in order to 
Table 1 Modules, objectives and topics of MIPAS-Family Program child training (each session $90 \mathrm{~min}$ )

\begin{tabular}{|c|c|c|}
\hline No of session/ modules & Objectives & Topics \\
\hline $\begin{array}{l}\text { 1. Group session } \\
\text { Module Ia: Clinical } \\
\text { features of headaches }\end{array}$ & We learn about headaches! & Interactive exploration of headaches: "We check the headache" \\
\hline $\begin{array}{l}\text { 2. Group session } \\
\text { Module Ib: The cause } \\
\text { of headache }\end{array}$ & We learn to understand the headaches (cause)! & $\begin{array}{l}\text { Collect knowledge; explanation of migraine and TTH; } \\
\text { identification of stress and sensory stimuli: "We are headache } \\
\text { experts!" }\end{array}$ \\
\hline $\begin{array}{l}\text { 3. Group session } \\
\text { Module IIa: Coping } \\
\text { with headaches }\end{array}$ & We learn to cope with headaches! & $\begin{array}{l}\text { Introduction to PMR; differential and conditioning relaxation; } \\
\text { "We learn the tricks to keep cool and relax!" }\end{array}$ \\
\hline $\begin{array}{l}\text { 4. Group session } \\
\text { Module IIb: Lifestyle }\end{array}$ & $\begin{array}{l}\text { We learn to identify favorable versus unfavorable } \\
\text { lifestyles; we perceive stress situations and sensory } \\
\text { over stimulation }\end{array}$ & $\begin{array}{l}\text { Interactive exploration of stress and sensory conditions and } \\
\text { their link to body perceptions; stress and sensory induction, } \\
\text { body perception and relaxation: "We kill stress" }\end{array}$ \\
\hline $\begin{array}{l}\text { 5. Group session } \\
\text { Module IIIa: Stress and } \\
\text { Sensory Coping } \\
\text { Training I }\end{array}$ & $\begin{array}{l}\text { Confrontation and habituation of sensory stimuli; we } \\
\text { learn to cope with stress and sensory stimulation! }\end{array}$ & "Body check-exercise"; "The-Thick-Skin-Training” \\
\hline $\begin{array}{l}\text { 6. Group session } \\
\text { Module IIIb: Stress and } \\
\text { Sensory Coping } \\
\text { Training II }\end{array}$ & $\begin{array}{l}\text { Confrontation and habituation of sensory stimuli; we } \\
\text { learn to cope with stress and sensory stimulation! }\end{array}$ & $\begin{array}{l}\text { Counter conditioning; habituation training; "Do-not-make-me- } \\
\text { nervous -training" }\end{array}$ \\
\hline $\begin{array}{l}\text { 7. Group session } \\
\text { Module IIIc: Pain } \\
\text { Coping }\end{array}$ & We learn to cope with pain by self coping strategies! & $\begin{array}{l}\text { The } 10 \text { steps to coping with a migraine and "The Stop the Pain } \\
\text { Training" }\end{array}$ \\
\hline 8. Group session & Program review and transfer to daily situations & Learning to enjoy life \\
\hline
\end{tabular}

facilitate the parents becoming "co-trainers" who helped employ the techniques at home. Treatment, more specifically, involved something we term sensory coping training (SCT) for patients with migraine, along with a SMT for children suffering from tension-type headaches as well as a pain coping training (PCP) [31]. The SCT was deduced from fundamental etiopathogenetical findings supporting migraine as learning theory based and as a neurobiological disease [28, 32, 33]. The SCT consisted of the following steps: (a) identification and body-oriented perception of specific acoustic, visual and smell-processing stimuli, (b) learning of a progressive muscle relaxation (PMR) training, counter conditioning, and habituation processing. For the children, the training was called "The-Thick-Skin-Training" and "Do-Not-Make-Me-Nervous-Training". For a further description of the objectives and topics of the program, see Tables 1 and 2 .

\section{Description of the Biofeedback Program}

We simultaneously incorporated feedback of forehead electrical muscle activity (EMG) and hand skin temperature (Thermal Biofeedback), along with other strategies (identification of psychophysiological relationships, relaxation, etc.) described in the literature [35]. During biofeedback, each child was encouraged to reduce the individualized EMG activity (baseline) up to defined training criteria of $20 \%$. That means that criteria for reduction of the EMG activity were individually determined with respect to the baseline at the beginning of the each session. The criterion for success for thermal selfcontrol was defined as an increase of up to $3.5^{\circ} \mathrm{C}$ on the dominant hand of the child during each session. Each session was carried out once per week and lasted $50 \mathrm{~min}$. The training started with an exploration of the child's general state of health and a review of the headache diary (10 min). Then, the EMG electrodes at the frontal muscles and the thermistors were placed on the dominant hand following a 3-min adaptation/baseline period (3 $\mathrm{min})$ and a voluntary "self-control" condition during which the children attempted to produce the correct response but in the absence of feedback ( $2 \mathrm{~min})$. Next followed four feedback conditions ( 3 min each), with each being interrupted by a 2 min pause. The session ended with a final self-control condition ( $2 \mathrm{~min}$ ) and a second baseline ( 3 min). The children were provided a small gift as reinforcement before leaving. Each child performed 20 sessions, for a total duration of $900 \mathrm{~min}$ of training. The biofeedback system was the Softmed (Insight Instruments, Vienna), which allows child-oriented feedback modalities (balloon and motorcycle rider) and specific reinforcement procedures. 
Table 2 Modules, objectives and topics of the MIPAS-Family parental training; (each session $120 \mathrm{~min}$ )

\begin{tabular}{|c|c|c|}
\hline No of session/modules & Objectives & Topics \\
\hline $\begin{array}{l}\text { 1. Parental group session } \\
\text { Module I }\end{array}$ & $\begin{array}{l}\text { Learn to identify and to understand headaches and their } \\
\text { causes }\end{array}$ & $\begin{array}{l}\text { Interactive exploration with respect to the child's } \\
\text { headache; collecting knowledge about headaches; } \\
\text { explanation of facts about headache (diagnoses, } \\
\text { classification, causes, medication); introduction to } \\
\text { stress and sensory diaries }\end{array}$ \\
\hline $\begin{array}{l}\text { 2. Parental group session } \\
\text { Module II }\end{array}$ & $\begin{array}{l}\text { Realizing the parental role in maintenance of headache; } \\
\text { educational style; the role of stress coping and sensory } \\
\text { over stimulation }\end{array}$ & $\begin{array}{l}\text { Analyze a parent-child interaction video; interactive } \\
\text { exploration of a specific stress and sensory situation } \\
\text { for each child; first stress and sensory induction and } \\
\text { body perception exercises; ten suggestions for } \\
\text { reducing stress }\end{array}$ \\
\hline $\begin{array}{l}\text { 3. Parental group session } \\
\text { Module III a }\end{array}$ & $\begin{array}{l}\text { Introduction and practice of stress and sensory coping } \\
\text { training (SCT) to be transferred to parental home; } \\
\text { learning of the relaxation training (PMR) for the } \\
\text { whole family }\end{array}$ & $\begin{array}{l}\text { Performance of PMR with parents (differential and } \\
\text { conditioned relaxation); stress coping and sensory } \\
\text { coping training; Habituation training ("Thick-Skin- } \\
\text { Training"; "Do-Not-Make-Me-Nervous-Training") }\end{array}$ \\
\hline $\begin{array}{l}\text { 4. Parental group session } \\
\text { Module III b }\end{array}$ & $\begin{array}{l}\text { Become acquainted with medication; learning the pain } \\
\text { coping training for home practice }\end{array}$ & $\begin{array}{l}\text { Understand the safe application of medication in } \\
\text { children; the role of negative reinforcement; practice } \\
\text { of the ten steps of the pain coping training of the } \\
\text { children }\end{array}$ \\
\hline
\end{tabular}

\section{Dependent measures}

\section{Headache and social activity diary}

To evaluate the efficacy of the intervention programs, the children were asked to complete a combined headache and social activity diary, at the end of each day, in which they tracked the headache parameters of frequency, duration (in hours), intensity, and medications consumed, as well as daily living activities (school attendance, homework, and leisure time). Children were asked to maintain these diaries for 4 weeks prior to, during, and after the intervention was completed. However, only $40 \%$ of the children were fully compliant with this request.

\section{Headache questionnaire}

The parents and the children were asked to complete a headache questionnaire, which was a German adaptation of the Pain Relevant Response Scale (PRRS). This is a modified version of a scale designed to assess the extent to which significant others respond to pain behavior [36, 37]. The 18-item scale asked parents and children to indicate how often they would attend to headache intensity using a visual analog scale (VAS, from 0 to 10), frequency (by a scale from 1 to 5 , whereas $1=$ daily headache, $2=$ several times per week, $3=$ once per week, $4=1-3$ times per week, $5=$ once the month), and duration (in hours per week), assist in treatment (i.e., offer a massage, offer medication, try to distract), and/or suggest or allow a reduction in activity (i.e., go to bed, dispense with chores, skip school). Higher scores reflect more frequent and more varied responses to pain.

\section{Quality of life (KINDL)}

The children were asked to complete the KINDL ${ }^{\circledR}$ prior to and during the follow-up assessments. This questionnaire measures health-related QoL in children and adolescents, and can be completed by children as well as the parents [38, 39]. The KINDL has been tested in a representative sample of German children and adolescents as well as their parents. The resulting psychometric measures provide reference values, which extend the potential of the $\mathrm{KINDL}^{\circledR}$ questionnaire and show a good reliability, as well as satisfactory validity, and a high level of acceptance [37]. In our study, we only used the version $\mathrm{KINDL}^{\circledR}$ self-rating which is composed of 24 items (Likert scale) related to the following six dimensions: Physical Well-Being, Psychological Well-Being, Self-Esteem, Family, Friend, and Everyday Functioning (school). Scores from these six dimensions can be summed to yield a Total Score. The $\mathrm{KINDL}^{\circledR}$ sub-scales as well as the $\mathrm{KINDL}^{\circledR}$ Total Score allow a quantification of the health-related QoL from the point of view of the children.

\section{Data analysis}

Our power analysis determined that approximately 50 participants would be required per cell in an optimal design. However, as this was determined to be an exploratory investigation and an extensive amount of time was 
required for treating each participant, we targeted 20 participants per group. During treatment, five patients' withdrew due to problems with adherence: one from the MIPAS-Family group and four from the Biofeedback group. The parent and child data were subjected to separate two-way ANOVAs (condition $\times$ time).

The analysis of the child headache diaries included the following steps: at first data (e.g., the duration of attacks in 4 weeks) assessed during the baseline were labeled as $100 \%$. So if a child during the 4 -week baseline period suffered in total $150 \mathrm{~h}$, this duration was defined as $100 \%$. If the child after the treatment suffered from $75 \mathrm{~h}$ of headache, we would say the headache frequency was reduced by $50 \%$. Equally, the values of the discrepancy were specified as group average values of these percentages. These values finally were calculated by inferential statistics. For the estimation of the general effectiveness additionally effect strength computations were accomplished by Cohen's $d$ for 6- and 12-month follow-up [40].

\section{Results}

Child headache diaries

Figure 1 shows percentage improvement for the different headache parameters as well as the social activities for both treatment groups (baseline vs. intervention period). We found a significant reduction in the headache intensity $($ Biofeedback $=47 \% ; \quad$ MIPAS-Family $=27 \%)$ but no significant improvement in the headache duration and frequency.

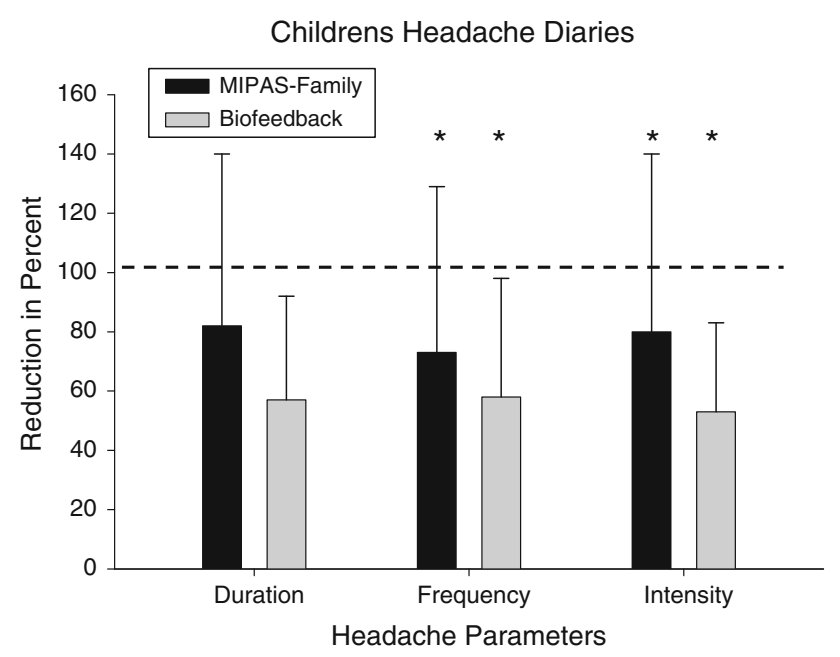

Fig. 1 Changes in mean percentages related to the baseline (4 weeks prior to the interventions; $100 \%$, broken line) in the parameters headache duration, frequency and intensity for both groups compared to the intervention period (mean values and standard deviations; ${ }^{*} p<.05$; explanation: the smaller the bar, the greater the effect)
The ANOVA for headache intensity showed a significant main effect for period $\left(F_{(1,27)}=15.534, p<.001\right)$, but not however for the main effect of group $\left(F_{(1,27)}=2.773, p<.107\right)$ or the interaction of group by time $\left(F_{(1,27)}=0.562, p<.460\right)$. In addition, the parameter headache intensity revealed a significant main effect for the course $\left(F_{(1,27)}=7.632, p<.01\right)$, but not for the factors interaction $\left(F_{(1,27)}=1.219, \quad p<.279\right)$ and group $\left(F_{(1,27)}=0.008, \quad p<.931\right)$. The parameter headache duration on the other hand showed no significant main effects or an interaction effect (course: $F_{(1,27)}=1.874$, $p<.185$; interaction: $F_{(1,27)}=2.742, p<.109$; group: $\left.F_{(1,27)}=1.705, p<.203\right)$. It must be kept in mind that the reports of the children might be less valid because a high standard deviation was found.

Reports from the parents' and children's headache questionnaires

Table 3 shows the statistical values for both intervention groups, the headache symptoms (duration, intensity and frequency) and comparing parents' and children's reports with each other. The statistical analyses mostly revealed a significant effect for time, with no group or interaction (group/time) effect. Thus, both groups showed an improvement in headache symptoms over time.

\section{Headache duration}

The Wilcoxon test demonstrated a significant difference between the Pre and both Post comparisons with respect to the parental reports on headache duration in the MIPASFamily group (Pre-Post $1: z=-2.44, p=.015$; Pre-Post 2: $z=-2.176, p=.03)$. No significant difference was found in the Post 1-Post 2 comparison. Likewise, only the comparison of Pre-Post conditions $(z=-2.092$, $p=.036$ ) based on child report (MIPAS-Family group) revealed a significant reduction in the headache duration.

In the Biofeedback group, no significant differences with respect to headache duration across the different measurement points were found.

To examine whether the experimental and control group reports (child and parent report) differed significantly in their levels of headache duration at Pre, Post 1 and Post 2, a Mann-Whitney test was conducted. No significant differences between both groups across the different measurement points were revealed. Due to the equality of the covariance matrices revealed in the Box test, an ANOVA could not be computed.

The parents reported a reduction in the headache duration on average from $4.74 \mathrm{~h}(\mathrm{SD}=2.8)$ in the beginning of the study to $3.24 \mathrm{~h}(\mathrm{SD}=1.9)$ directly after the study (Post 1) and down to $3.17 \mathrm{~h}(\mathrm{SD}=1.53)$ for Post 2 . The 
Table 3 Parent and child questionnaire ratings for headache duration (hours/week), frequency $(1=$ daily headache, $2=$ several times per week, $3=$ once per week, $4=1-3$ times per week, $5=$ once per month), and mean intensity for both conditions (MIPAS MIPASFamily, BFT Biofeedback), prior to and following treatment

\begin{tabular}{|c|c|c|c|c|c|c|c|c|c|c|c|c|}
\hline \multirow[t]{2}{*}{ Parameters } & \multicolumn{4}{|l|}{ Pre } & \multicolumn{4}{|c|}{ Post $1-6$ months } & \multicolumn{4}{|c|}{ Post $2-12$ months } \\
\hline & $M$ & SD & $\mathrm{MD}$ & $N$ & $M$ & SD & $\mathrm{MD}$ & $N$ & $M$ & $\mathrm{SD}$ & $\mathrm{MD}$ & $N$ \\
\hline \multicolumn{13}{|l|}{ Parents } \\
\hline \multicolumn{13}{|c|}{ Duration in hours/weekly } \\
\hline MIPAS & 4.75 & 2.80 & 4.25 & 19 & 3.24 & 1.90 & 3.00 & 19 & 3.17 & 1.53 & 3.00 & 16 \\
\hline BFT & 12.00 & 10.07 & 9.50 & 15 & 10.92 & 9.60 & 8.00 & 15 & 8.11 & 9.69 & 4.00 & 12 \\
\hline \multicolumn{13}{|l|}{ Frequency } \\
\hline MIPAS & 2.74 & 0.87 & 2.00 & 19 & 3.37 & 1.26 & 4.00 & 19 & 3.88 & 1.02 & 4.00 & 16 \\
\hline BFT & 2.00 & 1.07 & 2.00 & 15 & 2.73 & 1.53 & 2.00 & 15 & 2.92 & 1.62 & 2.50 & 12 \\
\hline \multicolumn{13}{|l|}{ Intensity } \\
\hline MIPAS & 3.81 & 2.49 & 4.00 & 19 & 2.55 & 1.88 & 3.00 & 19 & 2.44 & 2.70 & 2.00 & 16 \\
\hline BFT & 4.63 & 1.90 & 5.00 & 15 & 3.40 & 2.03 & 3.00 & 15 & 4.09 & 1.92 & 4.00 & 12 \\
\hline \multicolumn{13}{|l|}{ Children } \\
\hline \multicolumn{13}{|c|}{ Duration in hours/weekly } \\
\hline MIPAS & 5.56 & 6.05 & 3.50 & 19 & 2.96 & 3.65 & 1.50 & 19 & 5.29 & 7.60 & 2.00 & 16 \\
\hline BFT & 10.07 & 9.32 & 8.00 & 15 & 9.60 & 10.60 & 3.00 & 15 & 11.75 & 11.91 & 7.50 & 12 \\
\hline \multicolumn{13}{|l|}{ Frequency } \\
\hline MIPAS & 2.42 & 1.07 & 2.00 & 19 & 3.61 & 1.14 & 4.00 & 18 & 3.63 & 1.09 & 4.00 & 16 \\
\hline BFT & 1.87 & 1.06 & 2.00 & 15 & 2.50 & 1.51 & 2.00 & 19 & 2.58 & 1.68 & 2.00 & 12 \\
\hline \multicolumn{13}{|l|}{ Intensity } \\
\hline MIPAS & 2.24 & 2.42 & 1.00 & 19 & 2.71 & 2.34 & 3.00 & 19 & 1.78 & 1.89 & 1.50 & 16 \\
\hline BFT & 3.27 & 2.24 & 3.00 & 15 & 2.40 & 2.85 & 1.00 & 15 & 2.71 & 2.26 & 2.50 & 12 \\
\hline
\end{tabular}

Mean $(M)$, standard deviation (SD), median (MD), and number of cases $(N)$ are displayed

children of this group also reported a mean reduction in the headache duration from Pre $(M=5.56, \mathrm{SD}=6.05)$ to Post $1(M=2.96, \mathrm{SD}=3.65)$, but an increase in Post 2 $(M=5.29, \mathrm{SD}=7.6)$.

\section{Headache frequency}

According to parent report in the MIPAS-Family group, the headache frequency decreased across the different measurement points. The Wilcoxon test showed significant differences both for the Pre-Post $1(p=.032)$ and for the Pre-Post $2(z=-2.443, p=.007)$ comparison. Similarly, the Biofeedback group revealed significant differences for the same comparisons (Pre-Post 1: $z=-2.142, p=.040$; Pre-Post 2: $z=-2.209, p=.027)$. In addition, the children of the MIPAS-Family group reported highly significant reductions of the headache frequencies (Pre-Post 1: $z=-2.841, \quad p=.004 ; \quad$ Pre-Post 2: $z=-3.126$, $p=.002$ ), whereas the children of the Biofeedback group only showed a significant improvement for the Pre-Post 1 comparison $(z=-2.333, p=.02)$. The ANOVA revealed a significant course effect $\left(F_{(1,27)}=19.283, p=.000\right)$ and a significant group effect $\left(F_{(1,27)}=4.722, p=.039\right)$ but no interaction effect.

\section{Headache intensity}

Headache intensity was assessed with a ten-level headache VAS. According to parent report in the MIPAS-Family group, a significant reduction of the headache intensity was only found for the Pre-Post 2 comparison $(z=-2.166$, $p=.03)$. The mean scores of VAS changed from Pre $M=3.75 \quad(\mathrm{SD}=2.44) \quad$ down to Post $1 \quad M=2.37$ $(\mathrm{SD}=2.37)$ and up to Post $2 M=2.6(\mathrm{SD}=1.64)$. No significant changes in the Biofeedback group were found. In this group, the VAS scores were: at Pre $M=3.37$ $(\mathrm{SD}=1.89)$, Post $1 M=3.07(\mathrm{SD}=2.31)$ and Post 2 $M=3.46(\mathrm{SD}=2.25)$. The ANOVA showed a significant interaction effect $\left(\right.$ group/course; $F_{(1,27)}=4.506, \quad p=$ $.045)$.

In the MIPAS-Family group, no significant alterations in the headache intensity were found according to child report (Pre: $M=4.26, \mathrm{SD}=2.3$; Post $1: M=3.87, \mathrm{SD}=2.6$; Post 2: $M=3.31, \mathrm{SD}=2.03$ ). However, in the Biofeedback group, significant differences were found across all measurement points (Pre-Post 1: $z=-2.694, p=.007$; Pre-Post 2: $z=-2.451, p=.014$; Post 1-Post 2: $z=$ $-2.014, p=.044)$. The mean VAS scores of the children decreased from Pre $M=5.23(\mathrm{SD}=1.41)$ to Post 1 
Table 4 Mean effect sizes (Cohen's $d$ ) for the three headache parameters recorded by parents and children for the 2 treatment conditions

\begin{tabular}{|c|c|c|c|c|}
\hline \multirow[t]{3}{*}{ Parameters } & \multicolumn{4}{|l|}{ Effect sizes $d$} \\
\hline & \multicolumn{2}{|c|}{ Pre-Post $1-6$ months } & \multicolumn{2}{|c|}{ Pre-Post 2-12 months } \\
\hline & MIPAS & Biofeedback & MIPAS & Biofeedback \\
\hline \multicolumn{5}{|l|}{ Parents } \\
\hline Duration & 0.63 medium & 0.11 insignificant & 0.70 medium & 0.39 small \\
\hline Frequency & 0.59 medium & 1.42 large & 1.20 large & 0.67 large \\
\hline Intensity & 0.40 small & 0.14 insignificant & 0.56 large & 0.54 large \\
\hline$N$ & 19 & 16 & 17 & 12 \\
\hline \multicolumn{5}{|l|}{ Children } \\
\hline Duration & 0.52 medium & 0.05 insignificant & 0.04 insignificant & 0.16 insignificant \\
\hline Frequency & 0.88 large & 0.49 small & 1.12 large & 0.51 large \\
\hline Intensity & 0.16 insignificant & 1.25 large & 0.38 small & 0.97 large \\
\hline$N$ & 19 & 15 & 16 & 12 \\
\hline
\end{tabular}

Effect sizes ranging from 0.20 to 0.50 are considered small, $0.50-0.80$ medium, and 0.80 and above large

$M=2.60(\mathrm{SD}=1.64)$ but elevated again up to $M=3.42$ $(\mathrm{SD}=2.23)$ at Post 2 . The ANOVA revealed a significant course effect $\left(F_{(1,27)}=11.090, p=.003\right)$.

Table 4 reveals effect size values as determined by Cohen's $d$, which are seen to range from insignificant to large, based on respondent and variable chosen. For example, the MIPAS-Family training led to better effects based on headache duration, whereas the Biofeedback group, at least from the view of the children, showed greater improvement for headache intensity.

QoL and daily activities

First, we analyzed the diaries of the children of both groups concerning their daily activities whereby we compared (by ANOVA) the intervention period with the baseline. Regarding the school attendance, the ANOVA showed a significant main effect for time $\left(F_{(1,27)}=4.081, p=.020\right)$, but no effects for the group factor $\left(F_{(1,27)}=1.032, p=\right.$ $.388)$ or interaction $\left(F_{(1,27)}=0.145, p=.697\right)$. A similar pattern of findings was revealed for homework and leisure activities: only significant effects for time (homework: $F_{(1,27)}=4.181, p=.020$; leisure: $F_{(1,27)}=7.081 ; p=$ $.010)$; however, no group effects (homework: $F_{(1,27)}=$ $0.493, p=.572$; leisure: $\left.F_{(1,27)}=0.008, p=.873\right)$ and interaction effects (homework: $F_{(1,24)}=0.460, p=.488$; leisure: $\left.F_{(1,27)}=0.009, p=.984\right)$ were found. In conclusion, both groups reported improvements of their everyday life activities across time.

Table 5 shows the statistical values of the KINDL $^{\circledR}$ Total Score and of the several different dimensions. The ANOVA of the Total Score of the QoL questionnaire KINDL $^{\circledR}$ revealed a significant main effect for time $\left(F_{(27,1)}=5.527, p=.029\right)$ but not for the group factor
$\left(F_{(27,1)}=0.120, p=.732\right)$ or interaction (group $\times$ time interaction) $\left(F_{(27,1)}=3.370, p=.08\right)$. Regarding the specific dimensions of the KINDL ${ }^{\circledR}$, which seem to be most relevant for headache, we found the following results: A significant effect for group $\left(F\left(_{1,27}\right)=4.908, p=.036\right)$ and time $\left(F_{(1,24)}=4.619, p=.041\right)$, but no interaction effect for the dimension Physical Well-Being was found. In addition, the Wilcoxon test revealed significant Post 1 to Post 2 improvements in the dimensions Psychological Well-Being $(z=2.388, p=.017)$ and Self-Esteem $(z=$ $2.266, p=.023)$. For all other dimensions, no significant effects were found. Thus, the improvements in QoL of the children were restricted mainly to the physical realm (see Table 5).

\section{Discussion}

The described results of the headache diary evaluations in our study indicate that both intervention groups showed a significant reduction of the headache symptomatology. In the Biofeedback group, the headache frequency during the treatment was on average reduced by about $40 \%$, which corresponded with the results by Rokicki et al. [41]. In the MIPAS-Family group, a smaller reduction of the headache frequency $(28 \%)$ was found. In a review of the literature of three decades, Penzien et al. [21, 42] estimate the mean improvement of psychological intervention up to $35-50 \%$.

Based on a meta-analysis, Hermann and Blanchard [22] concluded that the temperature and EMG Biofeedback training, in particular in combination with relaxation training, have been the most effective treatment strategies of headaches in childhood. Analyzing the Pre-Post results of the headache questionnaires, we found similarly 
Table 5 Quality of life scores $\left(\mathrm{KINDL}^{\circledR}\right)$, Total Score as well as the QoL dimensions for both groups (MIPAS MIPAS-Family, BFT Biofeedback), prior to and following treatment

\begin{tabular}{|c|c|c|c|c|c|c|c|c|c|c|c|c|}
\hline \multirow[t]{2}{*}{ Quality of life scores/dimensions } & \multicolumn{4}{|l|}{ Pre } & \multicolumn{4}{|c|}{ Post $1-6$ months } & \multicolumn{4}{|c|}{ Post $2-12$ months } \\
\hline & $M$ & SD & MD & $N$ & $M$ & SD & MD & $N$ & $M$ & SD & MD & $N$ \\
\hline \multicolumn{13}{|l|}{ Physical Well-Being } \\
\hline MIPAS & 9.68 & 3.06 & 9.00 & 19 & 9.74 & 3.41 & 9.00 & 19 & 9.06 & 3.21 & 8.50 & 16 \\
\hline BFT & 12.27 & 2.43 & 13.00 & 15 & 11.80 & 2.88 & 12.00 & 15 & 10.83 & 2.89 & 10.50 & 12 \\
\hline \multicolumn{13}{|l|}{ Psychological Well-Being } \\
\hline MIPAS & 7.00 & 1.68 & 7.00 & 18 & 7.68 & 2.54 & 7.00 & 19 & 7.50 & 2.39 & 7.00 & 16 \\
\hline BFT & 9.20 & 3.30 & 9.00 & 15 & 8.53 & 2.39 & 9.00 & 15 & 7.25 & 1.86 & 7.50 & 12 \\
\hline \multicolumn{13}{|l|}{ Self-Esteem } \\
\hline MIPAS & 10.74 & 2.86 & 11.00 & 19 & 10.11 & 2.23 & 10.00 & 19 & 9.27 & 2.89 & 9.00 & 15 \\
\hline BFT & 10.93 & 2.52 & 11.00 & 15 & 11.07 & 3.28 & 10.00 & 15 & 9.42 & 2.97 & 9.00 & 12 \\
\hline \multicolumn{13}{|l|}{ Family } \\
\hline MIPAS & 7.31 & 2.93 & 7.00 & 19 & 7.37 & 3.04 & 7.00 & 19 & 7.53 & 2.99 & 6.50 & 16 \\
\hline BFT & 7.73 & 2.37 & 8.00 & 15 & 7.53 & 1.96 & 7.00 & 15 & 7.42 & 2.19 & 6.50 & 12 \\
\hline \multicolumn{13}{|l|}{ Friends } \\
\hline MIPAS & 8.58 & 3.13 & 8.00 & 19 & 8.67 & 2.17 & 8.50 & 18 & 7.81 & 2.26 & 8.00 & 16 \\
\hline BFT & 8.73 & 2.94 & 8.00 & 15 & 9.40 & 2.87 & 10.00 & 15 & 8.58 & 3.23 & 8.50 & 12 \\
\hline \multicolumn{13}{|l|}{ Everyday Functioning (school) } \\
\hline MIPAS & 9.21 & 2.12 & 9.00 & 19 & 9.21 & 2.12 & 9.00 & 19 & 9.00 & 2.63 & 9.00 & 16 \\
\hline BFT & 9.60 & 2.53 & 10.00 & 15 & 9.60 & 2.53 & 10.00 & 15 & 8.67 & 2.39 & 8.00 & 12 \\
\hline \multicolumn{13}{|l|}{ Disease } \\
\hline MIPAS & 10.05 & 3.36 & 10.00 & 19 & 9.74 & 3.49 & 9.00 & 19 & 9.40 & 3.83 & 8.00 & 16 \\
\hline BFT & 9.80 & 2.04 & 10.00 & 15 & 8.93 & 2.55 & 9.00 & 15 & 9.10 & 2.60 & 8.50 & 12 \\
\hline \multicolumn{13}{|l|}{ Total Score } \\
\hline MIPAS & 64.61 & 11.91 & 65.50 & 19 & 62.00 & 14.50 & 58.00 & 19 & 61.00 & 15.33 & 57.50 & 16 \\
\hline BFT & 71.00 & 11.31 & 72.00 & 15 & 66.87 & 11.24 & 72.00 & 15 & 60.20 & 11.56 & 57.00 & 12 \\
\hline
\end{tabular}

Mean $(M)$, standard deviation (SD), median (MD), and number of cases $(N)$ are displayed

significant reductions of headache duration, frequency and intensity, according to parent and child report. These results confirm former studies, especially regarding the Biofeedback training [17, 23]. Furthermore, the comparison of the effects of the combined Biofeedback training with the new multi-modal behavioral program MIPASFamily revealed that the multi-modal behavioral program produces similar clinical improvements than the individualized evidence-based medicine proven Biofeedback training [41, 43].

A comparable study was conducted by Dencke and Kröner-Herwig [43] which evaluated the effectiveness of EMG Biofeedback and relaxation training. Whether both treatments were more effective if parents took part in the treatment was investigated. Therefore, in one condition, parents participated in three sessions, whereas in another condition, children received one of the treatments alone. Fifty children, who suffered from migraine, tension headache or a combination of both, and their parents took part in the study. Using multivariate analysis, no significant group effect was found. However, a significant course effect was reported. A tendency was found that at the end of the training and 6 months later the Biofeedback training condition without relaxation and parental inclusion showed better results $[4,43]$.

Our study solely could demonstrate the general effectiveness of a multi-modal behavioral training; however, it does not allow any conclusions about the specific effectiveness of the parental training module. In a randomized study, Allen and Shriver [44] investigated whether an additional education of the parents (pain behavior management guidelines) could positively influence the effect of temperature Biofeedback training in 27 children suffering from headaches. One group obtained Biofeedback training (BFB) and the other group Biofeedback and education of the parents $(\mathrm{BFB}+\mathrm{OP})$. The authors found that the children in both groups experienced significant reductions in the headache activity. In addition, the BFB + OP group experienced significantly better outcomes across all headache parameters and a greater improvement in adaptive functioning for up to 3 months after the training compared to the BFB group. However, the significant differences of 
both groups disappeared during 1-year follow-up after the treatment [45]. Hence, this study cannot answer the question concerning the specific role parents play in the effectiveness of the treatment of their children. But the clinical observations of the authors and the concluding remarks that parents generally complied with the pain behavior management recommendations could be confirmed by our experiences. We found that, with proceeding session, parents were increasingly motivated to participate in the training. During the training session, we realized how little many parents know about the headaches of their children. This fact seems to be an explanation for the under estimation of the child's headache severity we found in the headache questionnaire. We agree with Allen and Shrine [44] that it is important to inform parents that their own behavior may exacerbate the chronicity of their child's pain.

An interesting study submitted by Mérelle et al. [46] evaluated the efficacy of a lay trainers program in adult patients with migraine. The objectives of the training resemble the ones in our parental training. Based on a structural manual, the lay trainers obtained a specific education to implement the objectives of the behavioral training in seven 2-h sessions. The authors found that 6 months after the training, in $42 \%$ of the patients, a $50 \%$ improvement was found. They concluded that attack frequency and QoL remained significantly (modestly) improved and feelings of control and self-confidence remained strongly improved [46]. Though this study included adult patients it could be assumed that parents as lay trainers could support the therapeutic processes. Further studies should be directed to a comparison of parental lay training with a professional child training.

We were furthermore interested whether Biofeedback or multi-modal approaches have differential indications for specific types of headache characteristics. The calculation of the effect sizes revealed high mean effect sizes in the headache frequencies for all comparisons as well as for the evaluations of the parents and the children. However, we found higher effect sizes for the MIPAS-Family group in the parameter headache duration, but on the other side higher effect sizes for the Biofeedback group in the parameter headache intensity. The extent of the effect sizes corresponds with results of former studies. In a new metaanalysis, which included 53 studies concerning Biofeedback in pediatric headaches $(N=10)$ and adults $(N=43)$, Nestoriuc et al. [20] concluded that Biofeedback is more effective than solely monitoring headaches, placebo and relaxation training. In the Biofeedback group, they found the highest symptom-specific effect sizes for the Pre-Post comparisons in the headache frequency $(d=0.82)$ and intensity $(d=0.69)$ [20]. The findings indicated that Biofeedback in pediatric headaches seems to be more effective than in adults and is more effective than other treatment approaches. These estimations confirm a former metaanalysis by Trautmann et al. [19] which found that headache intensity was the main efficacy criteria in pediatric headaches.

Finally, an interesting side effect of our study concerns the direct comparison of the headache evaluations of parents and the children. We found that parents generally overestimated the headache symptomatology of their children. As expected, the highest congruence in parentchild report was found for the parameter "headache frequency".

The present study again demonstrated that a combined Biofeedback training is effective and, apart from the symptomatology, also could improve the children's QoL. However, due to economical reasons (time and money), the similar effectiveness of the MIPAS-Family group program could be an interesting alternative to individualized trainings, such as Biofeedback. Whereas the MIPAS-Family group training is more time consuming for the whole family (in total $20 \mathrm{~h}, 8 \mathrm{~h}$ for the parental training and $12 \mathrm{~h}$ for the child-training compared to $16.7 \mathrm{~h}$ for the child only in the biofeedback group) it is less time-consuming for the therapist because it takes place in the group context in which at least eight to ten children can be treated at one time. Therefore, the MIPAS-Family group training is less expensive for the health care system than 20 individual sessions of Biofeedback training. Furthermore, even though each session in the MIPAS-Family group takes more time $(4 \times 120 \mathrm{~min}$ for parents and $8 \times 90 \mathrm{~min}$ for children), fewer sessions are needed (12 compared to 20 Biofeedback sessions).

Besides the economical reasons, MIPAS-Family training gives children with pediatric headache the opportunity to learn in a social context how to cope with their pain. This has the advantage that the children learn that there are other children with the same or similar complaints; this could be experienced as social support. In addition, they can learn from each other, in the group context, how to cope with the pain. As we already mentioned, we realized how little parents know about the headaches of their children. Therefore, we find it highly important to involve the parents in treatment in which they learn how to support their children in pain management. This might improve family cohesion and family functioning and can be seen as another social support factor for the child [47]. Furthermore, in the MIPAS-Family training, exercises can be incorporated in the daily activities of child and family which make it more likely that the learning processes can be transferred in everyday life.

The small number of patients in the study has to be regarded as the main limitation. Furthermore, the groups differed in some variables prior to the interventions. The 
lack of compliance of the children to complete the headache diaries after training was regrettable. However, despite these limitations, we conclude that, similar to other behavioral prevention programs (i.e., diabetes, asthma), the presented MIPAS-Family Program seems to be an effective intervention approach for pediatric headaches.

Conflict of interest None.

\section{References}

1. Wang SJ (2003) Epidemiology of migraine and other types of headache in Asia. Curr Neurol Neurosci Rep 3:104-108

2. Fendrich K, Vennemann M, Pfaffenrath V, Evers S, May A, Berger K, Hoffmann W (2007) Headache prevalence among adolescents - the German DMKG headache study. Cephalalgia 27:347-354

3. Unalp A, Dirik E, Kurul S (2007) Prevalence and clinical findings of migraine and tension-type headache in adolescents. Pediatr Int 49(6):943-949

4. Winner P (2008) Classification of pediatric headache. Curr Pain Headache Rep 12(5):357-360

5. Oelkers-Ax R, Resch F (2002) Kindliche Kopfschmerzen als Problem für die Kinder- und Jugendlichen Psychiatrie? Z Kinder Jugendpsychiatr Psychother 30(4):281-293

6. Hershey AD, Powers SW, Vockell AL, LeCates SL, Segers A, Kabbouche (2004) Development of a patient-based grading scale for PedMIDAS. Cephalalgia 24(10):844-849

7. Talarska D, Zgorzalewicz-Stachowiak M (2007) The influence of selected factors on the quality of life of children with headaches. Adv Med Sci 2(52 Suppl 1):123-125

8. Bandell-Hoekstra I, Abu-Saad HH, Passchier J, Knipschild P (2000) Recurrent headache, coping, and quality of life in children: a review. Headache 40(5):357-370

9. Bandell-Hoekstra IE, Abu-Saad HH, Passchier J, Frederiks CM, Feron FJ, Knipschild P (2002) Coping and quality of life in relation to headache in Dutch schoolchildren. Eur $\mathrm{J}$ Pain 6(4):315-321

10. Frare M, Axia G, Battistella PA (2002) Quality of life, coping strategies, and family routines in children with headache. Headache 42(10):953-962

11. Nodari E, Battistella PA, Naccarella C, Vidi M (2002) Quality of life in young Italian patients with primary headache. Headache 42(4):268-274

12. Bussone G, Usai S, Grazzi L, Rigamonti A, Solari A, D'Amico D (2004) Disability and quality of life in different primary headaches: results from Italian studies. Neurol Sci 25(Suppl 3):105107

13. Powers SW, Patton SR, Hommel KA, Hershey AD (2004) Quality of life in paediatric migraine: characterization of agerelated effects using PedsQL 4.0. Cephalalgia 24(2):120-127

14. Hershey AD (2005) What is the impact, prevalence, disability, and quality of life of pediatric headache? Curr Pain Headache Rep 9(5):341-344

15. Ferrari A, Pileri F, Spaccapelo L, Zappaterra M, Ruggerini C, Tacchi R, Bertolini A (2009) How children and parents evaluate the headache centre's intervention. Headache 49:194-201

16. Vannatta K, Getzoff EA, Powers SW, Noll RB, Gerhardt CA, Hershey AD (2008) Multiple perspectives on the psychological functioning of children with and without migraine. Headache 48(7):994-1004
17. Andrasik F, Powers SF, McGrath PJ (2005) Methodological considerations in research with special populations: children and adolescents. Headache 45:520-525

18. Holroyd KA, Drew JB (2006) Behavioral approaches to the treatment of migraine. Semin Neurol 26(2):199-207

19. Trautmann E, Lackschewitz H, Kröner-Herwig B (2006) Psychological treatment of recurrent headache in children and adolescents-a meta-analysis. Cephalalgia 26(12):1411-1426

20. Nestoriuc Y, Rief W, Martin A (2008) Meta-analysis of biofeedback for tension-type headache: efficacy, specificity, and treatment moderators. J Consult Clin Psychol 76(3):379-396

21. Penzien DB, Rains JC, Andrasik F (2002) Behavioral management of recurrent headache: three decades of experience and empiricism. Appl Psychophys Biofeedback 27(2):163-181

22. Hermann C, Blanchard EB (2002) Biofeedback in the treatment of headache and other childhood pain. Appl Psychophys Biof 27(2):143-162

23. Hermann C, Kim M, Blanchard EB (1995) Behavioral and prophylactic pharmacological intervention studies of pediatric migraine: an exploratory metaanalysis. Pain 60:239-256

24. Larsson B, Carlsson J, Fichtel A, Melin L (2005) Relaxation treatment of adolescent headache sufferers: results from a schoolbased replication series. Headache 45(6):692-704

25. Nestoriuc Y, Martin A (2007) Efficacy of biofeedback for migraine: a meta-analysis. Pain 128(1-2):111-127

26. McGrath PJ, Cunningham SJ, Lascelles MA, Humphreys $P$ (1990) Help yourself. A treatment for migraine headaches. University of Ottawa Press, Ottawa

27. Dencke H, Kröner-Herwig B (1998) Therapie des Kopfschmerzes im Kindesalter [Treatment of paediatric headaches]. Med Rep 22:17

28. Kröner-Herwig B, Mohn U, Pothmann R (1998) Comparison of biofeedback and relaxation in the treatment of pediatric headache and the influence of parent involvement of outcome. Appl Psychophys Biof 23:143-157

29. Kröner-Herwig B, Dencke H (2002) Cognitive-behavioral therapy of pediatric headache: are there differences in efficacy between a therapist-administered group training and a self-help format? J Psychosom Res 53(6):1107-1114

30. Siniatchkin M, Darabaneanu S, Gerber-von Müller G, Niederberger U, Petermann F, Schulte IE, Gerber WD (2010) Kinder mit Migräne und Asthma: Zur Rolle der Eltern-kind Interaktion [Children with migraine and asthma: the role of the parent-child interaction]. Kindheit und Entwicklung 19:27-35

31. Gerber WD, Stephani U, Kirsch E, Kropp P, Siniatchkin M (2002) Slow cortical potentials in migraine families are associated with psychosocial factors. J Psychosom Res 5:215-222

32. Gerber WD, Petermann F, Gerber-von Müller G, Niederberger U, Rentmeister B, Siniatchkin M, Stephani U (2008) MIPAS-Family-Entwicklung und Evaluation eines verhaltensmedizinisches Programms zur Behandlung kindlicher Kopfschmerzen [MIPAS-Development and evaluation of a behavioral program for paediatric headaches]. Verhaltenstherapie 18:43-48

33. Gerber WD, Schoenen J (1998) Biobehavioral correlates in migraine: the role of hypersensitivity and information-processing dysfunction. Cephalalgia 18(Suppl 21):5-11

34. Gerber WD, Kropp R, Schoenen J, Siniatchkin M (1996) „Born to be wild oder doch gelernt?" Neue verhaltensmedizinische Erkenntnisse zur Ätiopathogenese der Migräne ["Born to be wild or learned?" New behavioral medicine findings in the etiopathogenesis of migraine]. Verhaltenstherapie 6:10-220

35. Arndorfer RE, Allen KD (2001) Extending the efficacy of a thermal biofeedback treatment package to the management of tension-type headaches in children. Headache 41:183-192

36. Andrasik F, Schwartz MS (2006) Behavioral assessment and treatment of pediatric headache. Behav Modif 30(1):93-113 
37. Kerns RD, Rosenberg R (1995) Pain-relevant responses from significant others: development of a significant-other version of the WHYMPI scales. Pain 61:245-249

38. Bullinger M, Bruett AL, Erhart M, Ravens-Sieberer U, the BELLA Study Group (2008) Psychometric properties of the KINDL-R questionnaire: results of the BELLA study. Eur Child Adolesc Psychiatry 17:125-132

39. Ravens-Sieberer U (2001) The revised $\mathrm{KINDL}^{\circledR}$ : final results on reliability, validity and responsiveness of a modular HRQOL instrument for children and adolescents. Qual Life Res 10(3): 199

40. Cohen J (1992) A power primer. Psychol Bull 112:155-159

41. Rokicki LA, Holroyd KA, France CR, Lipchik GL, France JL, Kvaal SA (1997) Change mechanisms associated with combined relaxation/EMG biofeedback training for chronic tension headache. Appl Psychophys Biof 22(1):21-41

42. Rains JC, Penzien DB, McCrory DC, Gray RN (2005) Behavioral headache treatment: history, review of the empirical literature, and methodological critique. Headache 45(2):92-109
43. Dencke H, Kröner-Herwig B (2000) Kopfschmerz-Therapie mit Kindern und Jugendlichen: ein Trainingsprogramm [Headache Therapy with children and adolescents: a training program]. Göttingen, Hogrefe

44. Allen KD, Shriver MD (1998) Role of parent mediated pain behavior management strategies in biofeedback treatment of childhood migraines. Behav Ther 29:477-490

45. McGrath PA (1990) Commentary: psychological interventions for controlling children's pain: challenges for evidence-based medicine. J Pediatr Psychol 2:172-174

46. Mérelle SY, Sorbi MJ, van Doornen LJ, Passchier J (2008) Lay trainers with migraine for a home-based behavioral training: a 6month follow-up study. Headache 48(9):1311-1325

47. Gerber-von Müller G, Petermann U, Petermann F, Niederberger U, Stephani U, Siniatchkin M, Gerber Wd (2009) Das ADHSSummercamp. Entwicklung und Evalution eines multimodalen Programms [The ADHD-Summercamp. Development and evalution of multimodal program]. Kindheit und Entwicklung $18: 162-172$ 